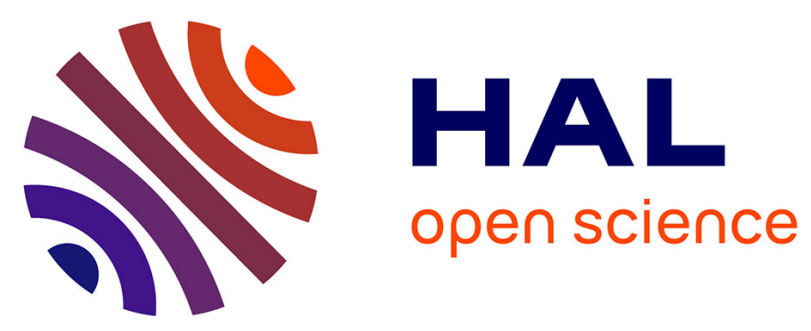

\title{
Tandem hydroformylation/isomerization/hydrogenation of bio-derived 1-arylbutadienes for the regioselective synthesis of branched aldehydes
}

\author{
G.M. Vieira, A.V. Granato, E.V. Gusevskaya, E.N. dos Santos, Pierre H.
}

Dixneuf, Cédric Fischmeister, Christian Bruneau

\section{To cite this version:}

G.M. Vieira, A.V. Granato, E.V. Gusevskaya, E.N. dos Santos, Pierre H. Dixneuf, et al.. Tandem hydroformylation/isomerization/hydrogenation of bio-derived 1-arylbutadienes for the regioselective synthesis of branched aldehydes. Applied Catalysis A: General, 2020, 598, pp.117583. 10.1016/j.apcata.2020.117583 . hal-02569952

HAL Id: hal-02569952

https://hal-univ-rennes1.archives-ouvertes.fr/hal-02569952

Submitted on 19 May 2020

HAL is a multi-disciplinary open access archive for the deposit and dissemination of scientific research documents, whether they are published or not. The documents may come from teaching and research institutions in France or abroad, or from public or private research centers.
L'archive ouverte pluridisciplinaire HAL, est destinée au dépôt et à la diffusion de documents scientifiques de niveau recherche, publiés ou non, émanant des établissements d'enseignement et de recherche français ou étrangers, des laboratoires publics ou privés. 


\section{Tandem hydroformylation/isomerization/hydrogenation of bio-derived 1- arylbutadienes for the regioselective synthesis of branched aldehydes}

Gabriel M. Vieiraa, Artur V. Granatoa, Elena V. Gusevskayaa, Eduardo N. dos Santosa*nicolau@ufmg.br, Pierre H. Dixneuf ${ }^{b}$, Cédric Fischmeister ${ }^{b}$, Christian Bruneau $^{\mathrm{b}^{*}}$ christian.bruneau@univ-rennes1.fr

a Departamento de Química - ICEx, Universidade Federal de Minas Gerais, Belo Horizonte, Brazil

b Univ Rennes, CNRS, ISCR (Institut des Sciences Chimiques de Rennes) - UMR6226, 35000 Rennes, France.

;

Graphical abstrqact

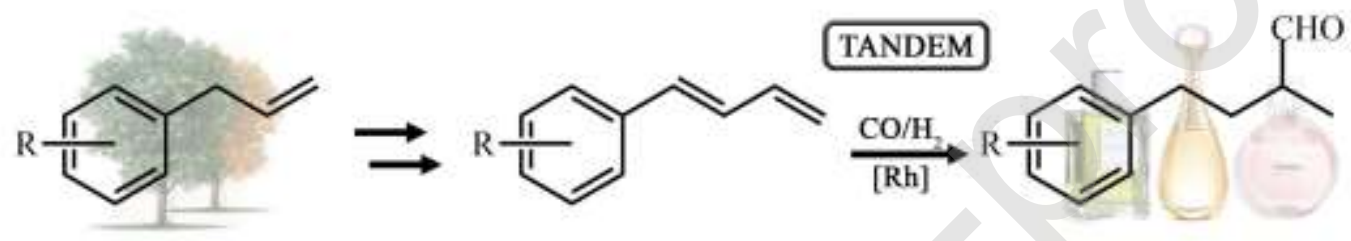

Highlights

- Synthesis of 1-arylbutadienes from bio-renewable 2-propenylbenzenes

- Tandem hydroformylation/isomerization/hydrogenation of 1-arylbutadienes

- Branched aldehydes useful as fragrances in high selectivity

\section{Abstract}

The rhodium-catalyzed hydroformylation of 1-arylbutadienes derived from lignocellulosic bio-resources has been carried out in toluene and green solvents. In the presence of DPPE and XANTPHOS ligands, a regioselective Markovnikov $\mathrm{Rh}-\mathrm{H}$ insertion takes place resulting in branched aldehydes in high selectivity, which contrasts with previous results obtained from aliphatic conjugated dienes. Depending on the nature of the diphosphine ligand, conjugated enals or saturated aldehydes are obtained in good to excellent selectivity. The later have a potential interest for fragrance industry, as they are homologous to commercial fragrance ingredients.

Keywords:

tandem catalysis, hydroformylation, fragrance

\section{Introduction}


Hydroformylation (oxo synthesis) is an efficient atom economical reaction, widely employed in industry, both in commodity and fine chemicals sector.[1] Formally, hydroformylation corresponds to the addition of a formyl group and a hydride to a $\mathrm{C}-\mathrm{C}$ double bond $(\mathrm{C}=\mathrm{C})$, as represented in Scheme 1 for an alphaolefin. The actual mechanism involves the combination of the olefin, carbon monoxide and hydrogen in the coordination sphere of a hydridocarbonyl transition metal complex. Although discovered long ago by Otto Rölen[2], this reaction still offers practical and scientific challenges because the selectivity control is highly dependent on the reactants, the catalytic system, and the reaction conditions.

\section{Insert Scheme 1}

State-of-art catalytic systems are predominately formed by a rhodium(I) precursor and phosphorus(III) ligands, and the design of new ligands has allowed to develop highly selective systems for specific applications.[3] For alpha-olefins, the linear product is preferentially formed for steric reasons, but high linear selectivity is only achieved by the employment of chelating phosphorus ligands with large bite angles.[4] Obtaining branched aldehydes in high selectivity when a methylidene group is in the alpha position of the $\mathrm{C}=\mathrm{C}$ is a far more complex task. We developed a catalytic system capable of producing the branched aldehyde in selectivity higher than $65 \%$ from naturally occurring 2propenylbenzenes based on a rhodium(I) pre-catalyst and the commercially available 1,3-bis(diphenylphosphino)propane (DPPP) as ancillary ligand.[5] More recently, by the employment of specially designed ligands, this regioselectivity was surpassed. [6-13] When the terminal $\mathrm{C}=\mathrm{C}$ bond has an electron-withdrawing group, a phenyl ring or a conjugated $\mathrm{C}=\mathrm{C}$ in the alpha position, the tendency to form branched products is higher. In this context, the hydroformylation of conjugated dienes opens opportunities for the synthesis of branched products, provided the selectivity can be controlled. The hydroformylation of 1,3-butadiene, isoprene and piperylene is useful for the synthesis of commodity products.[14-18] Also, 1,3-dienes moieties often occur in natural products, and their hydroformylation leads to aldehydes of interest for the fragrance industry.[19-21] However, hydroformylation of this kind of substrates is particularly difficult to achieve. Special reactions conditions, such as atypically high pressure, temperature or high ligand concentrations, are required to get reasonable reaction rates. Although there are more possibilities for the positioning of the formyl group, the primarily formed aldehydes in the hydroformylation of 1,3-dienes are usually the ones represented in Scheme $2[22]$.

Insert Scheme 2

Subsequent $\mathrm{C}=\mathrm{C}$ isomerization to form alpha,beta-unsaturated aldehydes followed by their hydrogenation are often observed, which leads to the problems of selectivity control. It is thus surprising that Nozaki et al. [23] were able to develop a system that allowed a regio and enantioselective hydroformylation of 1-phenylbutadiene and other dienes, although under rather harsh pressure conditions $\left(100\right.$ bar of $\left.\mathrm{CO} / \mathrm{H}_{2}\right)$, by the employment of (R)-2- 
(diphenylphosphino)-1,1-binaphthalen-2-yl(S)-1,1-binaphthalene-2,2-diyl phosphite ((R,S)-BINAPHOS) as a ligand. Years later, Landis et al. [22] employed chiral diazaphospholane ligands and developed a very efficient protocol that allows the regio and enantioselective hydroformylation of 1,3-dienes under much milder conditions. Although very efficient, these systems involve noncommercially available ligands whose synthesis is quite sophisticated. We considered that it would be worth to develop catalytic systems for the selective hydroformylation of conjugated dienes based on standard commercial ligands that could be readily implemented by industry. Thus, in this paper we describe a protocol to convert naturally occurring 2-propenylbenzenes[24], namely estragole, eugenol and safrole, to the corresponding 1-arylbutadienes, which were subsequently hydroformylated to produce in high selectivity novel branched aldehydes that can be useful for Food \& Flavor industry.

\section{Experimental}

The commercially acquired chemicals were used without an additional treatment except indicated cases. Estragole (98\%), eugenol (99\%), palladium acetate (99.9\%), triphenylphosphine (99\%), 1,2-bis(diphenylphosphino)ethane (98\%), 1,3-bis(diphenylphosphino)propane (97\%) and 9,9-dimethyl-4,5bis(diphenylphosphino)xanthene (98\%) were purchased from Acros Organics. Safrole (97\%), cesium carbonate (99\%), tris(2,4-di-tert-butylphenyl) phosphite (98\%) were purchased from Merck and dichloro(2-isopropoxyphenylmethylene) (tricyclohexylphosphine)ruthenium(II) (HGII) was supplied by Umicore. Tribenzylphosphine and dicarbonyl(2,4-pentanedionato)rhodium(I) (99\%) were purchased from Alfa Aesar. Allyl chloride (98\%) was purchased from Acros Organics, distilled under argon and stored over $3 \AA$ molecular sieves. Dichloromethane, acetonitrile and toluene were dried using MBraun solvent purification system.

Products were analyzed and characterized by GC and GC-MS using Shimadzu GC 2014 and QP2010-PLUS instrument (70 eV), respectively, both equipped with apolar column (Equity-5, $30 \times 0.25 \times 0.25)$. NMR spectra were recorded on a $400 \mathrm{MHz}$ Avance III spectrometer from Bruker. Chemical shifts are reported in ppm vs TMS using residual signals from deuterated solvents as internal reference.

\section{Preparation of the 1-arylbutadienes}

1-Arylbutadienes were prepared from naturally occurring 2-propenylbenzenes, namely, estragole (1a), eugenol (1b) and safrole (1c), using a previously reported route (Scheme 3).[25]

Insert Scheme 3

\section{Synthesis of 3-arylallyl chlorides (2a-c) (adapted from [25])}

For the synthesis of compound 2a, a Schlenk tube was loaded under argon with $1.0 \mathrm{~g}(0.0067 \mathrm{~mol})$ of estragole $(\mathbf{1 a}), 0.084 \mathrm{~g}(0.00013 \mathrm{~mol})$ of $2^{\text {nd }}$ generation Hoveyda-Grubbs (HGII), $3.1 \mathrm{~g}(0.040 \mathrm{~mol})$ of allyl chloride and $20 \mathrm{~mL}$ of 
dichloromethane. The mixture was refluxed for $15 \mathrm{~h}$. After evaporation of the solvent and the residual allyl chloride, the products were purified by column chromatography on silica gel using heptane/2\% ethyl acetate mixture as eluent. An analogous protocol was employed to synthesize compounds $\mathbf{2 b}$ and $\mathbf{2 c}$, but for the former a mixture heptane/20\% ethyl acetate was employed in the purification. The isolated yields are summarized in Table 1, the side products, if formed, were not characterized. The purified products were analyzed by ${ }^{1} \mathrm{H}$ and ${ }^{13} \mathrm{C}$ NMR, as well as GC-MS and the data are in Supporting Information S2.

\section{Synthesis of 1-arylbutadienes (3a-c) (adapted from [25])}

For the synthesis of compound 3a, a Schlenk tube was loaded under argon with $0.8 \mathrm{~g}(0.0041 \mathrm{~mol})$ of compound $2 \mathrm{a}, 0.046 \mathrm{~g}(0.000205 \mathrm{~mol})$ of palladium acetate, $0.107 \mathrm{~g}(0.00041 \mathrm{~mol})$ of triphenylphosphine, $1.59 \mathrm{~g}(0.0049 \mathrm{~mol})$ of cesium carbonate and $25 \mathrm{~mL}$ of acetonitrile. This reaction mixture was stirred under reflux for $17 \mathrm{~h}$. The resulting mixture was centrifuged at $6000 \mathrm{rpm}$ for $5 \mathrm{~min}$ and the supernatant was collected. The residual solid was washed with cold acetone, the mixture was centrifuged at $6000 \mathrm{rpm}$ for $5 \mathrm{~min}$ and the supernatant was collected. The supernatants were combined and the solvents were evaporated. The products were purified by column chromatography on silica gel using heptane $/ 2 \%$ ethyl acetate mixture as eluent. An analogous protocol was employed to synthesize compounds $\mathbf{3 b}$ and $\mathbf{3 c}$, but for the former a mixture heptane $/ 10 \%$ ethyl acetate was employed in the purification. . These products have already been described in the literature.[26, 27] The isolated yields are summarized in Table 1, the side products, if formed, were not characterized. The purified products were analyzed by ${ }^{1} \mathrm{H}$ and ${ }^{13} \mathrm{C}$ NMR, as well as GC-MS and the data are in Supporting Information S3-S4.

Insert Table 1

\section{Hydroformylation of 1-arylbutadienes (3a-c)}

The 1-arylbutadiene (3a) and phosphorus ligand were added in a $1.5 \mathrm{~mL}$ glass vial containing a magnetic stirrer. A solution of [ $\left.\mathrm{Rh}(\mathrm{acac})(\mathrm{CO})_{2}\right]$ in toluene was prepared in a Schlenk tube under argon atmosphere and the proper amount $(0.005 \mathrm{~mol})$ of $\mathrm{Rh}$ per $1.0 \mathrm{~mol}$ of starting material of catalyst solution was transferred to the glass vial containing the diene and closed with a cap containing a rubber septum pierced with a small needle. The vial was set in a stainless steel bomb, which was closed and purged with a $\mathrm{CO} / \mathrm{H}_{2}(1: 1)$ mixture. The bomb was then pressurized with 40 bar of this gas mixture and kept under magnetic stirring in an oil bath at the desired temperature $\left(60-100{ }^{\circ} \mathrm{C}\right)$ for 2 to $48 \mathrm{~h}$. The bomb was cooled and carefully vented in a hood and then opened to air. After solvent stripping, chloroform- $\mathrm{d}^{1}$ was added and the crude products were analyzed by ${ }^{1} \mathrm{H}$ NMR. The major products were separated by column chromatography on silica gel using heptane/ $2 \%$ ethyl acetate mixture as eluent. An analogous protocol was employed in the hydroformylation of compounds $\mathbf{3 b}$ and $3 c$, but for the former a mixture heptane $/ 20 \%$ ethyl acetate was employed in the purification of the main products. The purified products were analyzed by ${ }^{1} \mathrm{H}$ and ${ }^{13} \mathrm{C}$ NMR, as well as GC-MS and the data are in Supporting Information S4-S6. 


\section{Quantitative analysis of the hydroformylation products}

The quantification of the products was made by ${ }^{1} \mathrm{H}-\mathrm{NMR}$ of the crude reaction mixture. Depending on the catalytic system, up to 14 peaks were observed in the aldehyde region of the spectra ( $\delta 9.40-10.30 \mathrm{ppm})$, but only four of them were predominant. These major signals were attributed to compounds 4a, 5a, 6a and 7a. To the best of our knowledge, compounds $4 \mathbf{a}$ and $5 \mathbf{a}$ have not been reported before. They were isolated from the reaction solutions and fully characterized (see spectroscopic data below). Aldehydes 6a[28] and 7a[29] were previously described so that their aldehyde signals were attributed based on the literature data. The remaining peaks in the aldehyde region in the NMR spectra ( $\delta 9.40-10.30 \mathrm{ppm})$ of the crude product were added together and are reported in the Tables as "Others" (other aldehydes). The quantification was based on the intensities of the signals at $3.75-3.90 \mathrm{ppm}$ attributed to the methyl hydrogens of the methoxy groups present in all the products $(100 \%$ of the area $=$ $3 \mathrm{H})$. The mass balance was checked by the comparison of the sum integration of the aldehyde peaks $(1 \mathrm{H})$ and the methoxy peaks $(3 \mathrm{H})$.

\section{Results and Discussion}

\section{Hydroformylation of 1-arylbutadienes}

Estragole is the most available compound of the series and for this reason, the diene 3a derived from estragole was chosen as the substrate for hydroformylation in the initial studies. (Acetylacetonato)bis(carbonyl)rhodium(I), [Rh(acac)(CO)2], a widely used Rh(I) pre-catalyst in hydroformylation was used in combination with various phosphorus(III) ancillary ligands. Under the hydroformylation conditions, the precatalyst $\left[\mathrm{Rh}(\mathrm{acac})(\mathrm{CO})_{2}\right]$ is regarded to readily form in situ the 16-electron active catalyst $\left[\mathrm{RhH}(\mathrm{L})_{\mathrm{m}}(\mathrm{CO})_{\mathrm{n}}\right]$, in which $\mathrm{m}+\mathrm{n}=3$ and $\mathrm{L}$ is a phosphorus atom of the ancillary ligand.[30] We employed representative phosphorus(III) ligands of the class of monophosphines $\left(\mathrm{PPh}_{3}\right.$ and $\left.\mathrm{PBn}_{3}\right)$, monophosphite (TBDP) and chelating diphosphines with different bite angles (DPPE, DPPP, XANTPHOS), whose structures are depicted in Chart 1.

\section{Insert Chart 1}

The major products were $\mathbf{4 a}, \mathbf{5 a}, \mathbf{6 a}$ and $\mathbf{7 a}$ (Scheme 4). They differ from the generic ones in Scheme 2 because some of them are formed by consecutive reactions after hydroformylation, such as isomerization and hydrogenation. While linear aldehydes $\mathbf{6 a}$ and $\mathbf{7 a}$ can be formed directly by the hydroformylation of the terminal $\mathrm{C}=\mathrm{C}$ in $\mathbf{3 a}$, the formation of $\mathbf{4 a}$ should involve $\mathrm{a}$ further double bond migration that can occur either in the catalytic cycle or through a fast consecutive isomerization process of the $\beta, \gamma$-unsaturated branched aldehyde, which, nevertheless, was not observed by the analytical techniques employed. The hydrogenation of $\mathbf{4 a}$ gives the saturated branched aldehyde 5a. 


\section{Insert Scheme 4}

The first attempts for the hydroformylation of 3a were made employing the standard $\mathrm{PPh}_{3}$ as ancillary ligand at $100{ }^{\circ} \mathrm{C}, 40$ bar $-\mathrm{CO}: \mathrm{H}_{2}=1, \mathrm{P} / \mathrm{Rh}=10,24$ $\mathrm{h}$, but the substrate conversion was very low. Under harsher conditions $\left(100{ }^{\circ} \mathrm{C}\right.$, 80 bar $-\mathrm{CO}: \mathrm{H}_{2}=1, \mathrm{P} / \mathrm{Rh}=20,48 \mathrm{~h}$ ) it was possible to obtain a higher conversion (92\%), but the selectivity for individual aldehydes was very poor and other ligands were tested.

The results for the ligand screening in the hydroformylation of $\mathbf{3 a}$ are presented in Table 2. Employing the bulkier and more basic $\mathrm{PBn}_{3}$ (as compared to $\mathrm{PPh}_{3}$ ) as ancillary ligand, the substrate was completely converted after $24 \mathrm{~h}$ to give the saturated aldehyde $\mathbf{5 a}$ as a major product with $56 \%$ selectivity (Table 2 , entry 1). TBDP is a bulky phosphite known to be an efficient promoter in the rhodium-catalyzed hydroformylation of olefins.[31] Employing this compound as ancillary ligand under the same conditions also allowed a complete substrate conversion within $24 \mathrm{~h}$; however, the reaction was poorly selective providing a complex mixture of other aldehydes (Table 2, entry 2). As the data previously reported in the literature suggested that chelating ligands could give better results in the hydroformylation of conjugated dienes as compared to monodentated ligands[15], a set of chelating diphosphines with different bite angles was tested in the hydroformylation of 3a. The ligands employed and the corresponding bite angles were: DPPE $\left(85^{\circ}\right)$, DPPP $\left(91^{\circ}\right)$, and XANTPHOS $\left(108^{\circ}\right)$.[32] In the presence of DPPE, the ligand with the lowest bite angle in the series, not only a full conversion was obtained after $24 \mathrm{~h}$, but also a high selectivity (91\%) for the saturated aldehyde $\mathbf{5 a}$ (Table 2, entry 3). Thus, this system is very efficient for the tandem isomerization/hydroformylation/C=C hydrogenation, as observed previously by Behr et al.[18] employing isoprene as substrate. Nevertheless, 1-arylbutadiene gives the branched aldehyde, opposed to isoprene, which yields the terminal aldehyde. This is remarkable finding from the practical point of view, considering that such kind of branched aldehydes are difficult to obtain in high selectivity by the direct hydroformylation of alphaolefins.[6]

Insert Table 2

DPPP is a ligand with similar electronic features as DPPE, but with a larger bite angle. Although both systems presented the same trends, the selectivity of hydroformylation was lower in the system with DPPP (Table 2, cf. entries 4 and 3). [15] XANTPHOS is a bidentated ligand, well known to promote the rhodium-catalyzed hydroformylation of alpha-olefins with a high regioselectivity for linear aldehydes.[4] Thus, a higher selectivity for aldehydes 6a and 7a would be expected at the hydroformylation of 3a in the presence of XANTPHOS, but it was not actually observed (Table 2, entry 5). The branched aldehydes $\mathbf{4 a}$ and $\mathbf{5 a}$ were the major reaction products, with the combined selectivity being similar to that in the system promoted by DPPP (Table 2, cf. entries 4 and 5). It is noteworthy that there is a lower tendency to hydrogenate the $\alpha, \beta$-unsaturated aldehyde $\mathbf{4 a}$ by the XANTPHOS-promoted system, since even after $24 \mathrm{~h}, 37 \%$ of this aldehyde is present among the products. 
Considering that DPPE as ancillary ligand (L) gave the best results, further studies concerning the reaction conditions was carried out with this ligand and they are presented in Table 3. In entries 1-3, the effect of $\mathrm{L} / \mathrm{Rh}$ molar ratio can be observed. The systems with $\mathrm{L} / \mathrm{Rh}=2.5$ (entry 1 ) and 5.0 (entry 2) showed nearly the same performance, but at a higher ratio $(\mathrm{L} / \mathrm{Rh}=10$, entry 3 ) the contribution of linear aldehydes $\mathbf{6 a}$ and $\mathbf{7 a}$ in the product distribution increased. The effect of the reaction temperature can be evaluated by comparing the results presented in entries 2, 4, and 5 (Table 3). Although at $80{ }^{\circ} \mathrm{C}$ the conversion was completed after $24 \mathrm{~h}$, a significant amount of the branched aldehyde remained in the nonhydrogenated form (4a). This effect was even more evident in the reaction at 60 ${ }^{\circ} \mathrm{C}$, in which aldehyde $\mathbf{4 a}$ was a predominant product, thus clearly showing that higher temperature favored the hydrogenation step.

Insert Table 3

Although toluene is presently considered an acceptable solvent, there are some environmental issues assigned to this compound[33]. In a further study, we decided to test other solvents, with higher sustainability rankings than toluene, as a reaction medium for the hydroformylation of $\mathbf{3 a}$. $p$-Cymene has been successfully employed by us in hydroformylation[34] and metathesis.[35] For the system with $p$-cymene, the catalytic results were similar to those obtained in toluene (Table 3, cf. entries 2 and 6). Anisole has a much better sustainability rank than toluene and even than $p$-cymene,[33] and we have recently found it was an excellent solvent for hydroformylation[34]. Although the selectivity for aldehyde $\mathbf{5 a}$ in the reaction in anisole was slightly lower than in toluene, it was still reasonably high (85\% vs. 91\%, cf. entries 2 and 7 in Table $3)$. Thus, the more sustainable solvents $p$-cymene and anisole can be recommended as possible alternatives to toluene for the tandem hydroformylation/hydrogenation of conjugated diene 3a aiming at obtaining saturated aldehyde $\mathbf{5 a}$ with high selectivity.

In order to determine the reaction evolvement, a kinetic follow-up was performed and the results are presented in Figure 1. Under these reaction conditions, the substrate $\mathbf{3 a}$ is completely converted into products within $4 \mathrm{~h}$. The nearly straight line for the substrate consumption up to $100 \%$ conversion showed an independence of the reaction rate on the substrate concentration, thus suggesting saturation kinetics. After $2 \mathrm{~h}$, the predominant product was the unsaturated aldehyde $\mathbf{4 a}$, along with minor amounts of aldehydes $\mathbf{6 a}$ and $\mathbf{5 a}$. Then, the concentration of $\mathbf{4 a}$ started decreasing due to its conversion into $\mathbf{5 a}$, whereas the concentration of $\mathbf{6 a}$ continued to increase until $4 \mathrm{~h}$, and then decreased. The slight decrease of $\mathbf{6 a}$ until $24 \mathrm{~h}$ is probably due to the $\mathrm{C}=\mathrm{C}$ double bond isomerization to form other unsaturated aldehydes. Only after $24 \mathrm{~h}$ the formation of product $7 \mathbf{a}$ increased, likely due to the hydrogenation of $\alpha, \beta-$ unsaturated linear aldehydes rather than $\mathbf{6 a}$ directly. The variation in $\mathbf{5 a}$ percentage from 24 to $48 \mathrm{~h}$ (less than 4\%) is within the experimental reproducibility, although a decrease due to formation of minor amounts of nonaldehyde derivatives cannot be ruled out. The $\beta, \gamma$-unsaturated aldehyde 4a' isomer of $\mathbf{4 a}$ was not observed, but it would be the logic precursor of $\mathbf{4 a}$, firstly formed by the Markovnikov-type hydroformylation of the terminal double bond, followed by fast isomerization. Nevertheless, the direct formation of $\mathbf{4 a}$ as a 
primary product resulting from $1,3-\mathrm{H}$ migration in the coordination sphere of the metal within the catalytic cycle cannot be ruled out.[16]

Insert Figure 1

The substrate scope for the tandem hydrofomylation/hydrogenation process was extended to other 1-arylbutadienes derived from the natural products, eugenol (3b) and safrole (3c), being the results presented in Table 4.

Insert Table 4

The products, 4a-c and 5a-c obtained from these substrates are new compounds and the corresponding spectroscopic data are presented in the experimental section. As the diene double bonds of $\mathbf{3 a - 3 c}$ are conjugated to the aromatic ring, an influence of the ring substituents on the reaction output could be expected, being the more influent substituent at the para position. Nevertheless, the effect of the substituent of the aromatic ring was not so marked after $24 \mathrm{~h}$ for the systems promoted by DPPE (Table 4, entries 1-3). The high regioselectivity for the branched product $(4+5)$ was about the same for all three substrates. The difference in reactivity was more evident for the systems promoted by XANTPHOS. The hydroformylation rate was significantly lower for substrate $\mathbf{3 b}$ as compared to the other two as only $80 \%$ conversion was reached after $24 \mathrm{~h}$, whereas the product distributions were comparable (Table 4, entries 4, 5 and 6). Again, it was obvious that the XANTPHOS-promoted system was less efficient to promote the hydrogenation of $\mathbf{4}$ into $\mathbf{5}$, as $37-45 \%$ of $\mathbf{4}$ remained after $24 \mathrm{~h}$. At $60{ }^{\circ} \mathrm{C}$ (Table 4, entries 7 and 8) unsaturated aldehyde $4 \mathbf{a}$ accounted for $76 \%$ of the products at $62 \%$ conversion. Interestingly enough, if the reaction was prolonged to $48 \mathrm{~h}$ (entry 8 ) the conversion rose to $89 \%$, but no important change on product distribution occurred, which indicated that consecutive hydrogenation processes are not significant under these conditions. The unsaturated aldehyde $\mathbf{4 a}$ was thus obtained in $66 \%$ yield. It is noteworthy that the catalytic reaction can be driven selectively towards the unsaturated aldehyde $\mathbf{4 a}$ or the saturated aldehyde $\mathbf{5 a}$, simply by changing the reaction temperature and the auxiliary phosphorous ligand, DPPE or XANTPHOS, respectively.

Regarding to catalyst recovery and reuse, some of us published recently a methodology for the hydroformylation of 2-propenylbenzenes in which the catalyst is supported in an aqueous phase by means of the water-soluble sulfonated version of DPPE, inter alia.[36] The catalyst is recovered by decantation of the aqueous phase after hydroformylation. We believe that this system could be adapted for the 1-arylbutadienes 3a-c, and this should be a matter for further studies.

The results are fully consistent with the reaction paths shown in Scheme 5, based on Nozaki's proposal[23], which is supported by spectroscopic evidences. Although there would be other possibilities, the dominant interaction between 1-arylbutadienes 3 and the active rhodium species (LnRhH) occurs through the terminal C-C double bond. In Path A, the hydrogen is transferred to carbon in position 3 at the chain, leading to an Rh-alkyl species $\mathbf{A}$, which, after carbonylation and hydrogenolysis, yields aldehyde 6. Alternatively, path B, in which the hydrogen is transferred to carbon in position 4 at the chain, leads to 
the formation of a much more stable $\eta^{3}$-allyl intermediate $\mathbf{B}$, which seems to be the resting state for rhodium species and would explaining the observed saturation kinetics. The $\eta^{3}$-allyl intermediate $\mathbf{B}$ does not undergo directly carbonylation/hydrogenolysis, $[15,16,19,23]$ but it is in equilibrium with $\eta^{1}$ allyl intermediate $\mathbf{C}$, which can suffer carbonylation/hydrogenolysis. The primarily formed products can be further transformed by $\mathrm{C}=\mathrm{C}$ isomerization (migration) and hydrogenation. Under hydroformylation conditions catalyzed by rhodium, hydrogenation is only a major path when the $\mathrm{C}=\mathrm{C}$ double bond is conjugated with the aldehydic $\mathrm{C}=\mathrm{O}$, and thus the efficient hydrogenation occurring for the formation of $\mathbf{5}$ in high yield should be preceded by the formation of 4 . Likewise, the formation of $\mathbf{7}$ should also be preceded by the isomerization of 6 .

Insert Scheme 5

Although, the $\eta^{3}$-allyl (B) - $\eta^{1}$-allyl (C) equilibrium is widely favored towards the former, it can be shifted towards $\mathbf{C}$ by the enhancement of the steric encumbrance on rhodium, and this is the key point to explain the ligand effect.

Monodentated phosphorus ligands can promote the $\eta^{3}$-allyl- $\eta^{1}$-allyl shift by increase in their concentration or their binding ability. Increasing the concentration of certain ligands such as $\mathrm{PPh}_{3}$ may suffice in some cases,[16] but apparently the presence the conjugated phenyl ring in $\mathbf{B}$ makes it more stable, so that other ligands are needed. $\mathrm{PBn}_{3}$, a more basic and bulkier ligand than $\mathrm{PPh}_{3}$, acted better as a promoter in the hydroformylation of $\mathbf{3 a}$, probably, due to the shift of the $\eta^{3}-\eta^{1}$ equilibrium towards C. Employing TBDP as ancillary ligand, which is also bulky and binds strongly rhodium species, the same promoting effect on the transformation of $\eta^{3}$-intermediate $\mathbf{B}$ into $\eta^{1}$-intermediate $\mathbf{C}$ was observed. However, this ligand yields a more acidic rhodium intermediate, which is prone to promote the $\mathrm{C}$-C double bond isomerization and less prone to perform its hydrogenation. As a result, a complex mixture of isomeric aldehydes with low individual selectivity for each compound was formed when TBDP was employed as ligand.

Bidentated phosphines, such as DPPE, DPPP and XANTPHOS form more stable Rh species containing two coordinated phosphorus(III) due to the chelate effect. This forces the $\eta^{3}-\eta^{1}$ equilibrium to $\eta^{1}$ species (intermediate $C$, Scheme 5 ) and for that reason, diphosphines are more efficient for the hydroformylation of this kind of dienes than the corresponding monodentated ligands. DPPE is more efficient than DPPP because it forms more stable 5-membered ring chelates than the 6-membered ring of DPPP.[37].XANTPHOS has a wider bite angle and is conformationally more rigid than DPPE, resulting in a more encumbered environment around the $\mathrm{Rh}$ atom and favoring the formation of linear products in hydroformylation. [32] Thus, it is remarkable that the regioselectivity for the branched products $(\mathbf{4}+\mathbf{5})$ is comparable for both DPPE and XANTPHOS, since the latter is expected to favor Path A (less encumbered)[4] This finding reinforces the hypothesis that the regioselectivity in the hydroformylation of 1phenylbutadienes is biased by the formation of stable $\eta^{3}$-allyl $\mathbf{B}$, so that Path $B$ becomes a dominant reaction path, even in the presence of ligands know for directing hydroformilation for linear products. 
Although $\beta, \gamma$-unsaturated aldehyde 4' (Scheme 5) has not been detected in our work by the analytic technique used, its intermediate formation cannot be ruled out. On the other hand, the aldehydes similar to 4' were reported as major reaction products at the hydroformylation of related dienes with other catalytic systems and under different reaction conditions (in particular, lower temperatures in the range of $\left.30-40{ }^{\circ} \mathrm{C}\right) .[22,23]$ We suggest that in our systems, either the fast isomerization of $\beta, \gamma$-unsaturated aldehyde 4 ' into $\alpha, \beta$-unsaturated aldehyde 4 biased by the favorable conjugation of the $\mathrm{C}=\mathrm{C}$ and $\mathrm{C}=\mathrm{O}$ bonds is occurring, or the double bond migration starting from intermediate $\mathbf{C}$ to give directly aldehyde $\mathbf{4}$ is operating, as previously suggested.[16] Either way, under appropriate conditions, the $\alpha, \beta$-unsaturated aldehyde 4 can undergo hydrogenation in tandem to give branched aldehyde $\mathbf{5}$ in excellent yields. DPPE is the most efficient ancillary for this purpose. With XANTPHOS, the tandem hydrogenation is less efficient, probably, because the coordination of the trisubstituted $\mathrm{C}-\mathrm{C}$ double bond in $\mathbf{4}$ to the rhodium species is more difficult due to the greater steric encumbrance caused by this ligand. On the other hand, under appropriate temperature conditions, the use of the XANTPHOS-promoted system offers the possibility to obtain selectively another useful product, the $\alpha, \beta$-unsaturated aldehyde $\mathbf{4 a}$.

\section{Conclusions}

Bio-sourced 2-propenylbenzene derivatives have been transformed into 4-aryl-2-methylbutanals and 4-aryl-2-methylbutenals via a sequence of catalytic transformations involving ruthenium-catalyzed cross metathesis, palladiumcatalyzed dehydrochlorination and rhodium-catalyzed hydroformylation. The hydroformylation of the synthesized 1,3-dienes employing rhodium/monophosphorus ligands was slow and the selectivity was poor. Only by the employment of chelating diphosphines as ancillary ligands at relatively high concentration were the activity and selectivity increased. The selectivity was also highly dependent on the ligands. At $100^{\circ} \mathrm{C}$, consecutive C-C double bond isomerization and hydrogenation took place, resulting in a useful tandem process. Employing XANTPHOS as ancillary ligand, the C-C double bond hydrogenation was not efficient, allowing the synthesis of the intermediate enals 4a-c in good yields. Employing DPPE as ancillary ligand at $100{ }^{\circ} \mathrm{C}$, a clean tandem sequence took place: although this system was efficient to reduce the $\mathrm{C}$-C double bond of the enals, the C-O double bond remained untouched, leading to saturated branched aldehydes 5a-c in very good selectivity. These branched aldehydes are difficult to obtain through other routes and are potentially interesting for food and flavor industry as $\mathbf{5 a}$ and $\mathbf{5 c}$ are homologous to the commercial fragrances canthoxal and helional, respectively. Noteworthy is that these transformations could be carried out in green solvents such as anisole.

\section{CRediT author statement}

Gabriel M. Vieira (investigation), Artur V. Granato(investigation), Elena V. Gusevskaya (writing -Review \& Editing), Eduardo N. dos Santos (writingOriginal Draft, writing -Review \& Editing, supervision), Pierre H. Dixneuf 
(writing -Review \& Editing), Cédric Fischmeister(writing -Review \& Editing, supervision), Christian Bruneau(writing -Review \& Editing, supervision)

\section{Declaration of interests}

The authors declare that they have no known competing financial interests or personal relationships that could have appeared to influence the work reported in this paper.

\section{Acknowledgements}

The authors thank CAPES-COFECUB (project PHC 884-17-France; 883/2017-Brazil), INCT-Catalysis, and CNPq for financial support. Umicore is thanked for supplying Hoveyda-Grubbs catalyst M72 (C627).

\section{References}

[1] A. Borner, R. Franke, Hydroformylation: Fundamentals, Processes, and Applications in Organic Synthesis, Wiley-VCH, Weinheim, 2016.

[2] O. Rölen, Chem. Abstr., 38 (1944) 3631.

[3] E.V. Gusevskaya, J. Jimenez-Pinto, A. Borner, ChemCatChem, 6 (2014) 382411.

[4] P.W.N.M. van Leeuwen, P.C.J. Kamer, Catal. Sci. Technol., 8 (2018) 26-113.

[5] A.C. da Silva, K.C.B. de Oliveira, E.V. Gusevskaya, E.N. dos Santos, J. Mol. Catal. A Chem., 179 (2002) 133-141.

[6] G.M. Noonan, J.A. Fuentes, C.J. Cobley, M.L. Clarke, Angew. Chem. Int. Ed., 51 (2012) 2477-2480.

[7] P. Dingwall, J.A. Fuentes, L. Crawford, A.M.Z. Slawin, M. Buhl, M.L. Clarke, J. Am. Chem. Soc., 139 (2017) 15921-15932.

[8] L. Iu, J.A. Fuentes, M.E. Janka, K.J. Fontenot, M.L. Clarke, Angew. Chem. Int. Ed., 58 (2019) 2120-2124.

[9] V.F. Slagt, P.C.J. Kamer, P. W.N.M. van Leeuwen, J.N.H. Reek, J. Am. Chem. Soc., 126 (2004) 1526-1536.

[10] A. Phanopoulos, K. Nozaki, ACS Catal., 8 (2018) 5799-5809.

[11] T. Besset, D.W. Norman, J.N.H. Reek, Adv. Synth. Catal., 355 (2013) 348-352.

[12] P. Dydio, R.J. Detz, B. de Bruin, J.N.H. Reek, J. Am. Chem. Soc., 136 (2014)

8418-8429.

[13] X.W. Wang, S.S. Nurttila, W.I. Dzik, R. Becker, J. Rodgers, J.N.H. Reek, Chemi. Eur. J., 23 (2017) 14769-14777.

[14] H. Bahrmann, B. Fell, J. Mol. Catal., 8 (1980) 329-337.

[15] P.W.N.M. Van Leeuwen, C.F. Roobeek, J. Mol. Catal., 31 (1985) 345-353.

[16] H.J.V. Barros, C.C. Guimaraes, E.N. dos Santos, E.V. Gusevskaya, Organometallics, 26 (2007) 2211-2218.

[17] P. Neubert, S. Fuchs, A. Behr, Green Chem., 17 (2015) 4045-4052.

[18] A. Behr, S. Reyer, N. Tenhumberg, Dalton Trans., 40 (2011) 11742-11747.

[19] C.M. Foca, H.J.V. Barros, E.N. dos Santos, E.V. Gusevskaya, J.C. Bayon, New J. Chem., 27 (2003) 533-539.

[20] H.J.V. Barros, C.C. Guimaraes, E.N. dos Santos, E.V. Gusevskaya, Catal. Commun., 8 (2007) 747-750. 
[21] H.J.V. Barros, J.G. da Silva, C.C. Guimaraes, E.N. dos Santos, E.V. Gusevskaya, Organometallics, 27 (2008) 4523-4531.

[22] A.L. Watkins, C.R. Landis, Org. Lett., 13 (2011) 164-167.

[23] T. Horiuchi, T. Ohta, E. Shirakawa, K. Nozaki, H. Takaya, Tetrahedron, 53 (1997) 7795-7804.

[24] M. Hassam, A. Taher, G.E. Arnott, I.R. Green, W.A.L. van Otterlo, Chem.-Rev., 115 (2015) 5462-5569.

[25] H. Bilel, N. Hamdi, F. Zagrouba, C. Fischmeister, C. Bruneau, Catal. Sci.

Technol., 4 (2014) 2064-2071.

[26] M.H. Al-Huniti, M.A. Perez, M.K. Garr, M.P. Croatt, Org. Lett., 20 (2018) 73757379.

[27] T.Q. Wang, Y.Y. Hu, S.L. Zhang, Org. Biomol. Chem., 8 (2010) 2312-2315.

[28] Y. Jeong, D.Y. Kim, Y. Choi, J.S. Ryu, Org. Biomol. Chem., 9 (2011) 374-378.

[29] B. Meka, S.R. Ravada, M.K.K. Muthyala, P.N. Kurre, T. Golakoti, Bioor. Chem., 80 (2018) 408-421.

[30] R. Franke, D. Selent, A. Borner, Chem. Rev., 112 (2012) 5675-5732.

[31] H. Tricas, O. Diebolt, P.W.N.M. van Leeuwen, J. Catal., 298 (2013) 198-205.

[32] M.N. Birkholz, Z. Freixa, P. W.N.M. van Leeuwen, Chem. Soc. Rev., 38 (2009) 1099-1118.

[33] C.M. Alder, J.D. Hayler, R.K. Henderson, A.M. Redman, L. Shukla, L.E. Shuster, H.F. Sneddon, Green Chem., 18 (2016) 3879-3890.

[34] F.G. Delolo, E.N. dos Santos, E.V. Gusevskaya, Green Chem., 21 (2019) 10911098.

[35] A.V. Granato, A.G. Santos, E.N. dos Santos, ChemSusChem, 10 (2017) 18321837.

[36] P.J. Baricelli, M. Rodriguez, L.G. Melean, M.M. Alonso, M. Borusiak, M.

Rosales, B. Gonzalez, K.C.B. de Oliveira, E.V. Gusevskaya, E.N. dos Santos, Appl. Catal. A Gen., 490 (2015) 163-169.

[37] P. Dierkes, P.W.N.M. van Leeuwen, J. Chem. Soc. Dalton Trans., (1999) 15191529. 
Table 1 - Synthesis of 3-arylallyl chlorides (2a-c) and 1-arylbutadienes (3a-c)

\begin{tabular}{ccc}
\hline Starting material & Conversion (\%) & Isolated Yield (\%) \\
\hline 1a & Synthesis of 2a-c $\mathbf{c}^{\mathrm{a}}$ & \\
\hline 1b & 95 & $81(\mathbf{2 a})$ \\
1c & 85 & $71(\mathbf{2 b})$ \\
\hline 2a & 90 & $62(\mathbf{2 c})$ \\
\hline 2b & Synthesis of 3a-c ${ }^{\mathrm{b}}$ & $62(\mathbf{3 a})$ \\
$\mathbf{2 c}$ & 100 & $47(\mathbf{3 b})$ \\
\hline
\end{tabular}

a Conditions: 1a-c - $1.0 \mathrm{~g}$, allyl chloride (6 equiv.), HGII (2 mol\%), solvent - dichloromethane (20 $\mathrm{mL}), 40 \stackrel{\circ}{\circ}, 15 \mathrm{~h}$.

${ }^{\mathrm{b}}$ Conditions: 2a-c - 0.5-1.0 g, $\mathrm{Pd}(\mathrm{OAc})_{2}(5 \mathrm{~mol} \%), \mathrm{PPh}_{3}(10 \mathrm{~mol} \%), \mathrm{Cs}_{2} \mathrm{CO}_{3}$ (1.2 equiv.), solvent acetonitrile, $90^{\circ} \mathrm{C}, 17 \mathrm{~h}$.

Table 2 - Hydroformylation of $\mathbf{3 a}$ in the presence of different ligands ${ }^{\mathrm{a}}$

\begin{tabular}{cccccccc}
\hline \multirow{2}{*}{ Entry } & \multirow{2}{*}{ Ligand } & Conversion & \multicolumn{5}{c}{ Product distribution $(\%)$} \\
\cline { 4 - 8 } & $(\%)$ & $\mathbf{4 a}$ & $\mathbf{5 a}$ & $\mathbf{6 a}$ & $\mathbf{7 a}$ & Others $^{\mathbf{c}}$ \\
\hline $1^{\mathrm{d}}$ & PBn3 & 100 & 0 & 56 & 1 & 6 & 37 \\
$2^{\mathrm{d}}$ & TBDP & 100 & 0 & 21 & 0 & 3 & 76 \\
3 & DPPE & 100 & 0 & 91 & 3 & 0 & 6 \\
4 & DPPP & 100 & 2 & 73 & 7 & 2 & 16 \\
5 & XANTPHOS & 100 & 37 & 41 & 10 & 0 & 12 \\
\hline
\end{tabular}

a Conditions: 3a - 1.0 mol. $\mathrm{L}^{-1}$, [Rh(acac)(CO) 2 - $0.5 \mathrm{~mol} \%$; ligand - $2.5 \mathrm{~mol} \%$, solvent - toluene, $100 \stackrel{\circ}{\circ}$, 40 bar $\left(\mathrm{CO}: \mathrm{H}_{2}=1\right), 24 \mathrm{~h}$. ${ }^{\mathrm{b}}$ Determined by ${ }^{1} \mathrm{HNMR}$, zero value means not observed by the technique. ${ }^{c}$ Other products, mainly other aldehydes. ${ }^{\mathrm{d}}$ Ligand $-5.0 \mathrm{~mol} \%$. 
Table 3 - Hydroformylation of 3a using DPPE as ancillary under different reaction conditions $^{\mathrm{a}}$

\begin{tabular}{ccccccccc}
\hline \multirow{2}{*}{ Entry } & $\begin{array}{c}\text { Ligand } \\
(\text { mol \%) }\end{array}$ & $\begin{array}{c}T \\
\left({ }^{\circ} \mathrm{C}\right)\end{array}$ & $\begin{array}{c}\text { Conversion }^{\mathrm{b}} \\
(\%)\end{array}$ & \multicolumn{5}{c}{ Product distribution $^{\mathrm{b}}(\%)$} \\
\cline { 5 - 9 } & 1.25 & 100 & 100 & 0 & 87 & 3 & 0 & 9 \\
2 & 2.5 & 100 & 100 & 0 & 91 & 3 & 0 & 6 \\
3 & 5.0 & 100 & 100 & 0 & 83 & 5 & 6 & 6 \\
4 & 2.5 & 80 & 100 & 13 & 73 & 7 & 0 & 7 \\
5 & 2.5 & 60 & 91 & 54 & 35 & 3 & 0 & 8 \\
$6^{\mathrm{d}}$ & 2.5 & 100 & 100 & 0 & 88 & 4 & 3 & 5 \\
$7^{\mathrm{e}}$ & 2.5 & 100 & 100 & 0 & 85 & 6 & 3 & 6 \\
\hline
\end{tabular}

a Conditions: 3a - 1.0 mol. $\mathrm{L}^{-1}$; [Rh(acac)(CO) 2 - $0.5 \mathrm{~mol} \%$; solvent - toluene, 40 bar (CO: $\mathrm{H}_{2}=1$ ), $24 \mathrm{~h}$. ${ }^{\mathrm{b}}$ Determined by ${ }^{1} \mathrm{HNMR}$, zero value means not observed by the technique. ${ }^{\mathrm{c}}$ Other products, mainly other aldehydes. ${ }^{\mathrm{d}}$ Solvent - $p$-cymene. ${ }^{\mathrm{e}}$ Solvent - anisole.

Table 4 - Hydroformylation of 1-arylbutadienes ${ }^{\text {a }}$

\begin{tabular}{ccccccccc}
\hline \multirow{2}{*}{ Entry } & \multirow{2}{*}{ Ligand } & \multirow{2}{*}{ Substrate } & Conversion ${ }^{\mathrm{b}}$ & \multicolumn{5}{c}{ Product distribution $^{\mathrm{b}}(\%)$} \\
\cline { 6 - 9 } & & $\%$ & $\mathbf{4}(\mathbf{a}-\mathbf{c})$ & $\mathbf{5 ( a - c )}$ & $\mathbf{6}(\mathbf{a}-\mathbf{c})$ & $\mathbf{7 ( a - c )}$ & Others $^{\mathbf{c}}$ \\
\hline 1 & DPPE & $\mathbf{3 a}$ & 100 & 0 & 91 & 3 & 0 & 6 \\
2 & DPPE & $\mathbf{3 b}$ & 99 & 4 & 86 & 7 & 3 & 0 \\
3 & DPPE & $\mathbf{3 c}$ & 100 & 0 & 92 & 5 & 3 & 0 \\
4 & XANTPHOS & $\mathbf{3 a}$ & 100 & 37 & 41 & 10 & 0 & 12 \\
5 & XANTPHOS & $\mathbf{3 b}$ & 80 & 45 & 42 & 13 & 0 & 0 \\
6 & XANTPHOS & $\mathbf{3 c}$ & 100 & 45 & 46 & 9 & 0 & 0 \\
$7^{\mathrm{d}}$ & XANTPHOS & $\mathbf{3 a}$ & 62 & 76 & 3 & 12 & 0 & 9 \\
$8^{\mathrm{d}, \mathrm{e}}$ & XANTPHOS & $\mathbf{3 a}$ & 89 & 74 & 2 & 13 & 0 & 11 \\
\hline
\end{tabular}

a Conditions: substrate - 1.0 mol. $\mathrm{L}^{-1}$, [Rh(acac)(CO) 2$]-0.5 \mathrm{~mol} \%$, ligand - $2.5 \mathrm{~mol} \%$, solvent toluene, 40 bar (CO: $\left.\mathrm{H}_{2}=1\right), 24 \mathrm{~h}$. bDetermined by ${ }^{1} \mathrm{HNMR}$, zero value means not observed by the technique. ${ }^{c}$ Other products, mainly, other aldehydes. ${ }^{\mathrm{d}} 60^{\circ} \mathrm{C}$. e $48 \mathrm{~h}$. 


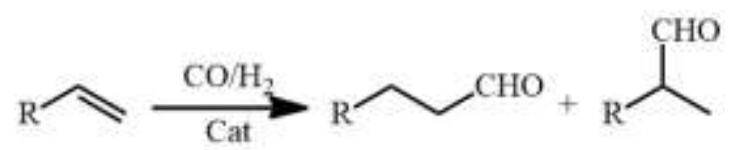

Scheme 1

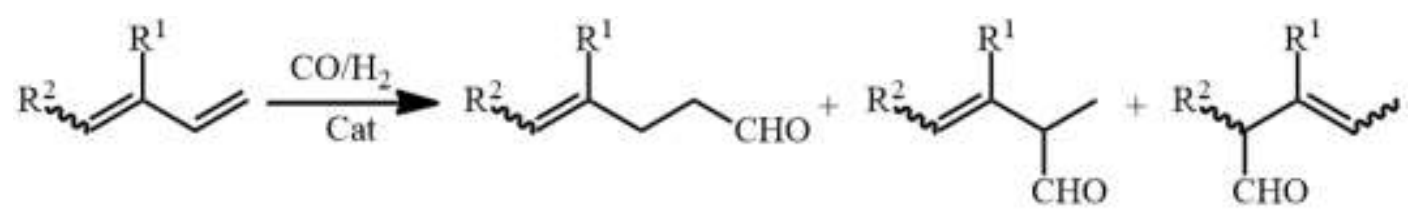

Scheme 2

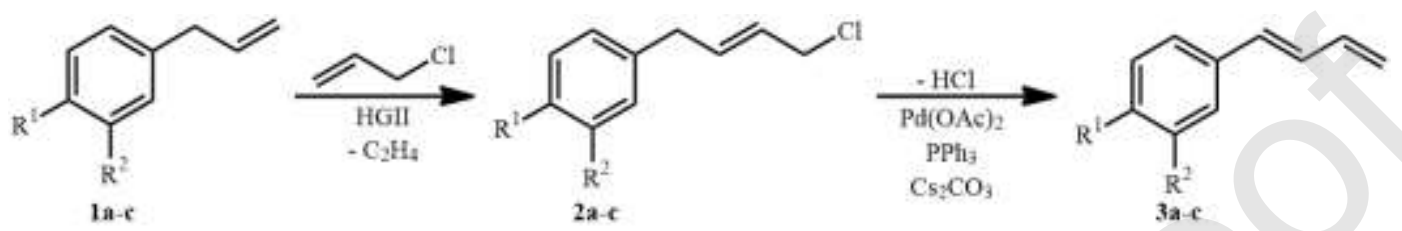

1-3a: $\mathrm{R}^{1}=\mathrm{OCH}_{3}, \mathrm{R}^{2}=\mathrm{H} \quad 1-3 \mathbf{b}: \mathrm{R}^{1}=\mathrm{OH}, \mathrm{R}^{2}=\mathrm{OCH}_{3} \quad 1-3 \mathrm{c}: \mathrm{R}^{1}-\mathrm{R}^{2}=-\mathrm{OCH}_{2} \mathrm{O}-$

Scheme 3

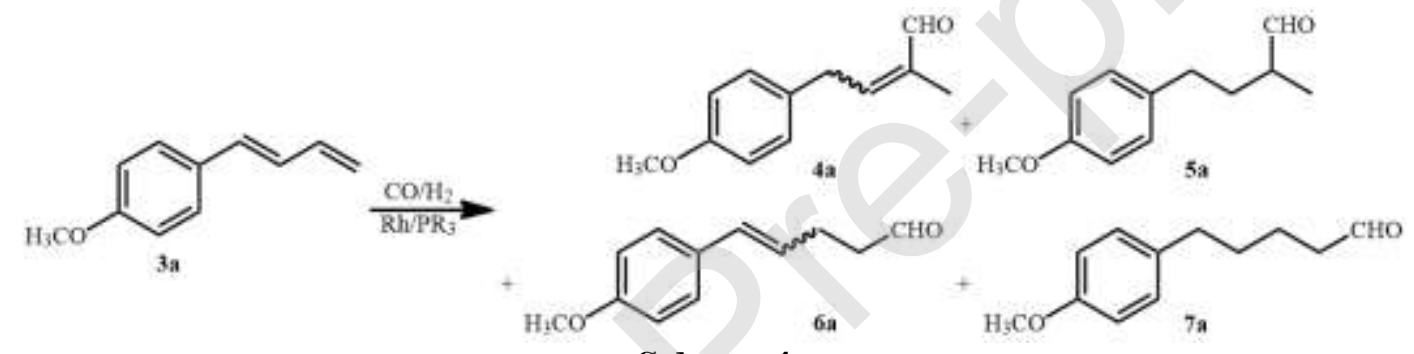

Scheme 4

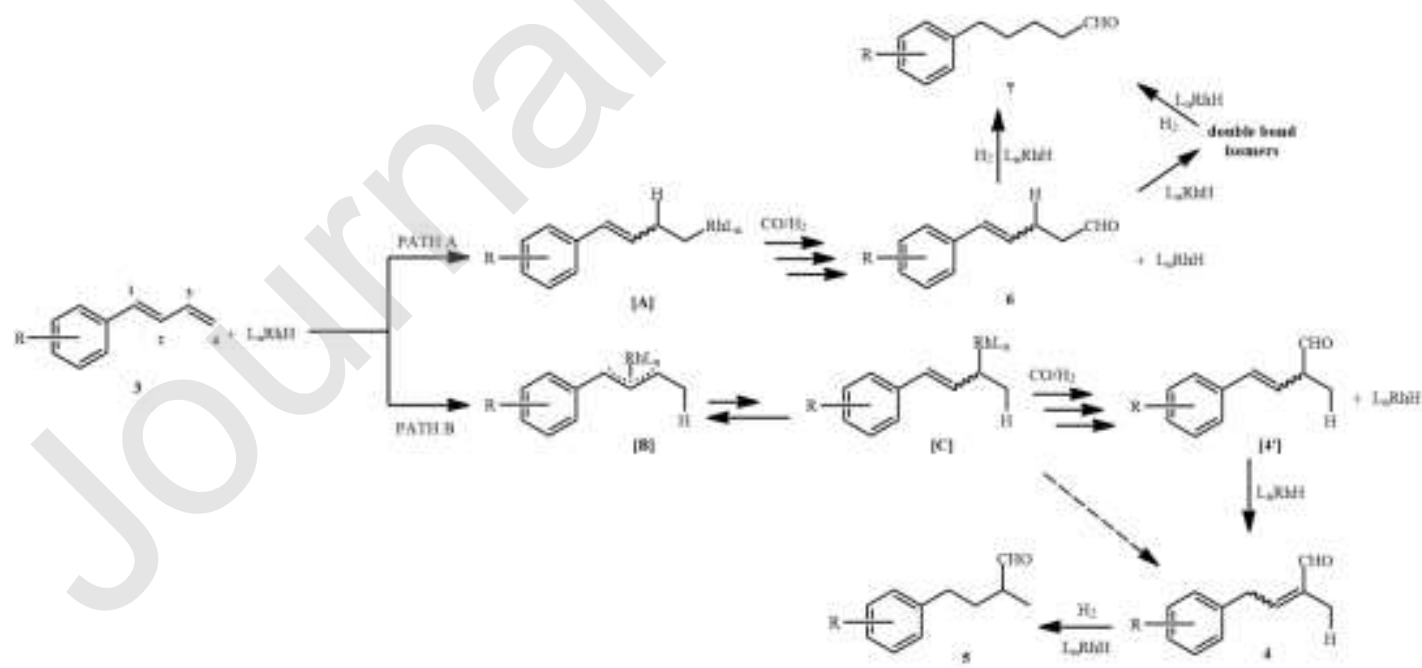

Scheme 5 


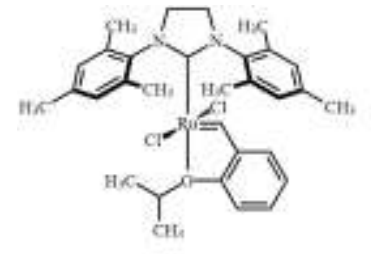

ngiा

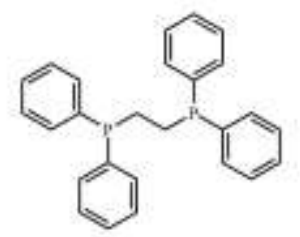

DPF.

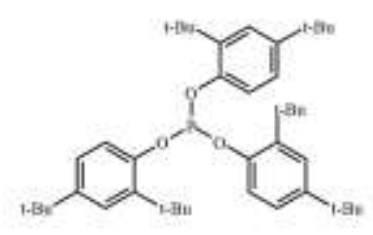

TEDP

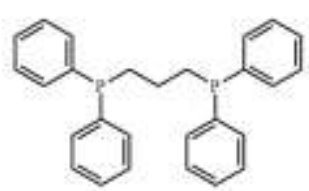

sppe<smiles>c1ccc(C(c2ccccc2)(c2ccccc2)c2ccccc2)cc1</smiles>

pros<smiles>c1ccc(CC(Cc2ccccc2)Cc2ccccc2)cc1</smiles>

rins

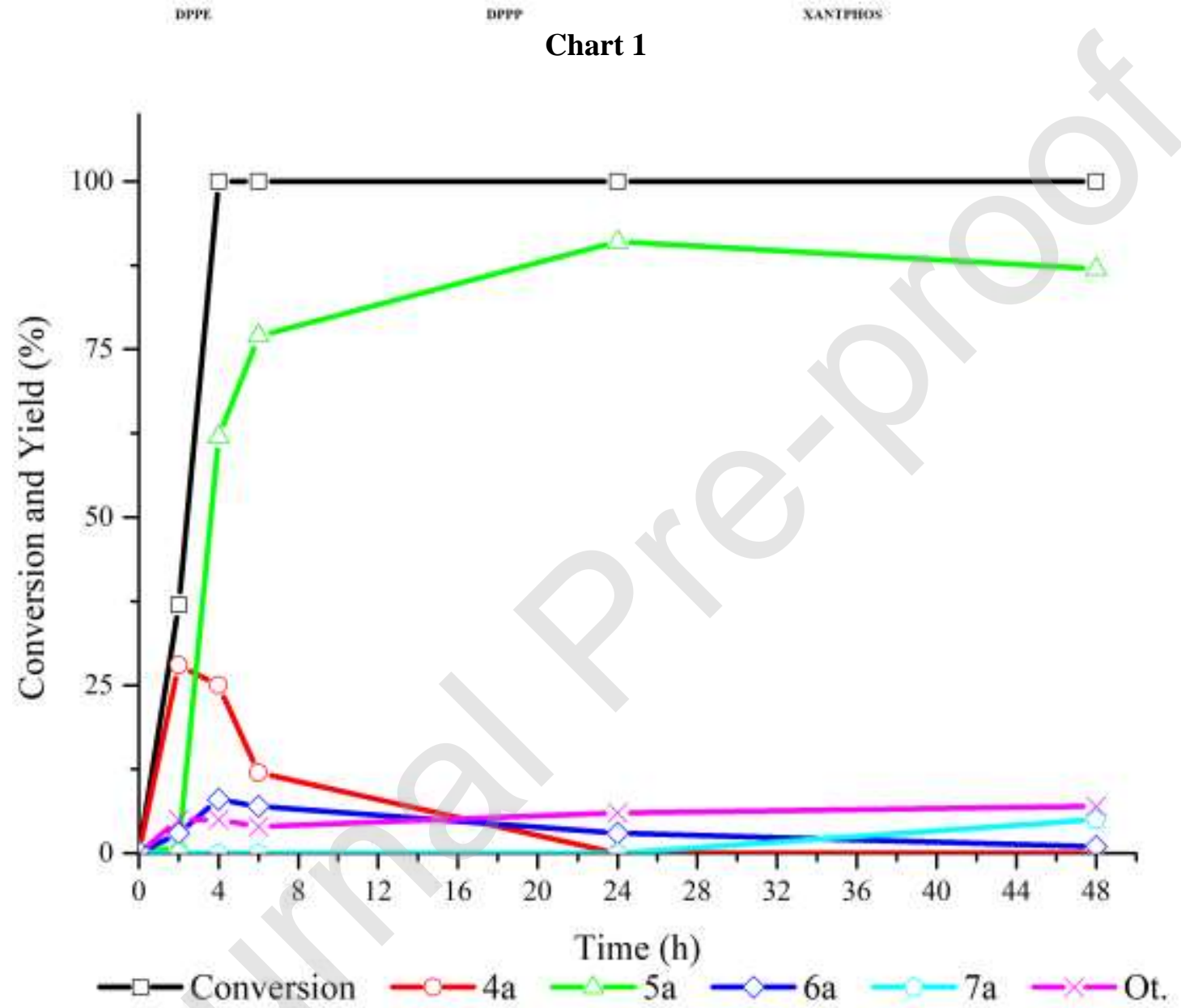

Figure 1: Kinetic follow-up for the hydroformylation of 3a promoted by Rh/DPPE. Conditions: $[3 \mathrm{a}]_{0}=1.0 \mathrm{~mol} . \mathrm{L}^{-1}$; $\left[\mathrm{Rh}(\mathrm{acac})(\mathrm{CO})_{2}\right], 0.5 \mathrm{~mol} \%$; DPPE, $2.5 \mathrm{~mol} \%$; toluene; $100^{\circ} \mathrm{C} ; 40$ bar $\left(\mathrm{CO}: \mathrm{H}_{2}=1\right) ; 24 \mathrm{~h} .{ }^{\mathrm{b}}$ determined by ${ }^{1} \mathrm{HNMR}$. Ot: Other products. 